\title{
Necrotizing Fasciitis of the Thigh Secondary to Radiation Colitis in a Rectal Cancer Patient
}

\author{
So Hyun Park, Jung Ran Choi, Ji Young Song, Kyu Keun Kang, Woong Sun Yoo, Sung Wan Han, \\ Choon Kwan Kim \\ Department of Infectious Diseases, Veterans Hospital Seoul Medical Center, Seoul, Korea
}

Necrotizing fasciitis usually occurs after dermal injury or through hematogenous spread. To date, few cases have been reported as necrotizing fasciitis of the thigh secondary to rectal perforation in rectal cancer patients. A 66-year-old male complained of pelvic and thigh pain and subsequently developed necrotizing fasciitis in his right thigh. Four years earlier, he had undergone a low anterior resection and radiotherapy due to of rectal cancer. An ulcerative lesion had been observed around the anastomosis site during the colonoscopy that had been performed two months earlier. Pelvic computed tomography and sigmoidoscopy showed rectal perforation and presacral abscess extending to buttock and the right posterior thigh fascia. Thus, the necrotizing fasciitis was believed to have occurred because of ulcer perforation, one of the complications of chronic radiation colitis, at the anastomosis site. When a rectal-cancer patient complains of pelvic and thigh pain, the possibility of a rectal perforation should be considered.

\section{Keywords: Radiation colitis; Necrotizing fasciitis; Rectal neoplasms; Thigh}

\section{INTRODUCTION}

Although necrotizing fasciitis is rare, 0.4 cases every 0.1 million adults, it is a serious life-threatening infectious disease which causes severe destructive necrosis in soft tissues and skin tissues [1]. In addition, as the mortality rate is very high even when the necrotic tissues are extensively resected, early diagnosis and intensive surgical treatment play important roles in reducing the mortality rate. However, early diagnosis is difficult because lesions are initiated in deep subcutaneous tissues and spread via the fascia without external clinical symptoms. This mainly takes place after the skin damage in the pelvic limb has occurred and sometimes occurs due to hematogenous spread as the result of secondary bacteremia.

Received: May 7, 2012 - Accepted: August 4, 2012

Correspondence to: Choon Kwan Kim, M.D.

Department of Infectious Diseases, Veterans Hospital Seoul Medical Center, 53 Jinhwangdo-ro 61-gil, Gangdong-gu, Seoul 134-791, Korea

Tel: +82-2-2225-1491, Fax: +82-2-2225-4348

E-mail: schenics@naver.com

(C) 2012 The Korean Society of Coloproctology

This is an open-access article distributed under the terms of the Creative Commons Attribution NonCommercial License (http://creativecommons.org/licenses/by-nc/3.0) which permits unrestricted noncommercial use, distribution, and reproduction in any medium, provided the original work is properly cited.
Necrotizing fasciitis can occur anywhere in the body; it commonly takes place in the limbs, but can also occur in abdominal walls, perianal regions, inguinal regions, and wounds caused by surgeries [2]. Only a couple of cases in which necrotizing fasciitis has occurred in femoral regions primarily without skin damage, including cases associated with direct perforation caused by colorectal cancers, have been reported [3-6]. Therefore, the authors, based on their experience, report the case of a patient with necrotizing fasciitis in femoral regions due to rectal perforation caused by rectal-cancer surgery and by complications of radiation therapy.

\section{CASE REPORT}

A 66-year-old male patient visited orthopedics due to pains in the right femoral regions and pelvis that had started a month earlier. No abnormal findings were during the diagnosis, including X-ray scanning etc. A week before, the patient had visited the emergency room as he was experiencing more severe pains in the right femoral regions and pelvis, as well as a fever that had started a day before. Four years earlier, the patient had received a lower anterior resection and a resection of the right lobe because of colorectal cancer and liver metastasis (American Joint Committee on Cancer stage T4N2M1, stage IV). After the surgery, adjuvant chemo- 
Journal of The Korean Society of A Case of Necrotizing Fasciitis of the Thigh Secondary to Radiation Colitis in a Rectal Cancer Patient

Coloproctology so Hyun Park, et al.

therapy was performed eight times with capecitabine and radiation therapy at 5,580 cGy; 31 fractions were carried out in the pelvis. Moreover, the patient was under medication due to diabetes and chronic obstructive pulmonary disease. No unusual findings were observed in the family history, and there was no history of drinking or smoking history. Vital signs at the time of his visit indicated $107 / 77 \mathrm{mmHg}, 90 / \mathrm{min}, 38.1^{\circ} \mathrm{C}$, and $18 / \mathrm{min}$ for blood pressure, pulse rate, body temperature, and respiration rate, respectively. No unusual findings were found on the chest auscultation, and the abdomen was soft without oppressive and rebound pains. No mass was palpated upon digital rectal examination. Severe pains in the right femoral regions and ion the side of the abdomen, as well as an erythematous rash, oppressive pains, and crepitus in the right femoral regions, were palpated in the patient. General blood tests showed leukocytes of $10,440 / \mathrm{mm}^{3}$, hemoglobin at $12.6 \mathrm{~g} / \mathrm{dL}$, a platelet count of $149,000 / \mathrm{mm}^{3}$, and an erythrocyte sedimentation rate of $50 \mathrm{~mm} / \mathrm{hr}$; C-reactive protein was elevated $(191 \mathrm{mg} /$ dL). General chemical tests resulted in a total protein content of $5.5 \mathrm{~g} / \mathrm{dL}$, albumin at $3.1 \mathrm{~g} / \mathrm{dL}$, total bilirubin at $1.0 \mathrm{mg} / \mathrm{dL}$, an aspartate aminotransferase/alanine aminotransferase ratio of 55/191 IU/L, alkaline phosphatase at $58 \mathrm{IU} / \mathrm{L}, \mathrm{r}$-glutamyl transpeptidase at $14 \mathrm{IU} / \mathrm{L}$, lactate dehydrogenase at $762 \mathrm{U} / \mathrm{mL}$, and a blood urea nitrogen/creatinine ratio of $16.1 / 0.6 \mathrm{mg} / \mathrm{dL}$; also, increases in creatine kinase (CK, 8,424 IU/L), CK-MB (20.14 ng/mL), and myoglobin $(1,325 \mathrm{ng} / \mathrm{mL})$ were noted. The $\mathrm{Na} / \mathrm{K} / \mathrm{Cl}$ ratio upon serum electrolyte examination was $130 / 3.7 / 96 \mathrm{mmol} / \mathrm{L}$.

The patient showed unique necrotizing fasciitis in the magnetic resonance imaging (MRI); a gluteus maximus muscle, a thick fas-

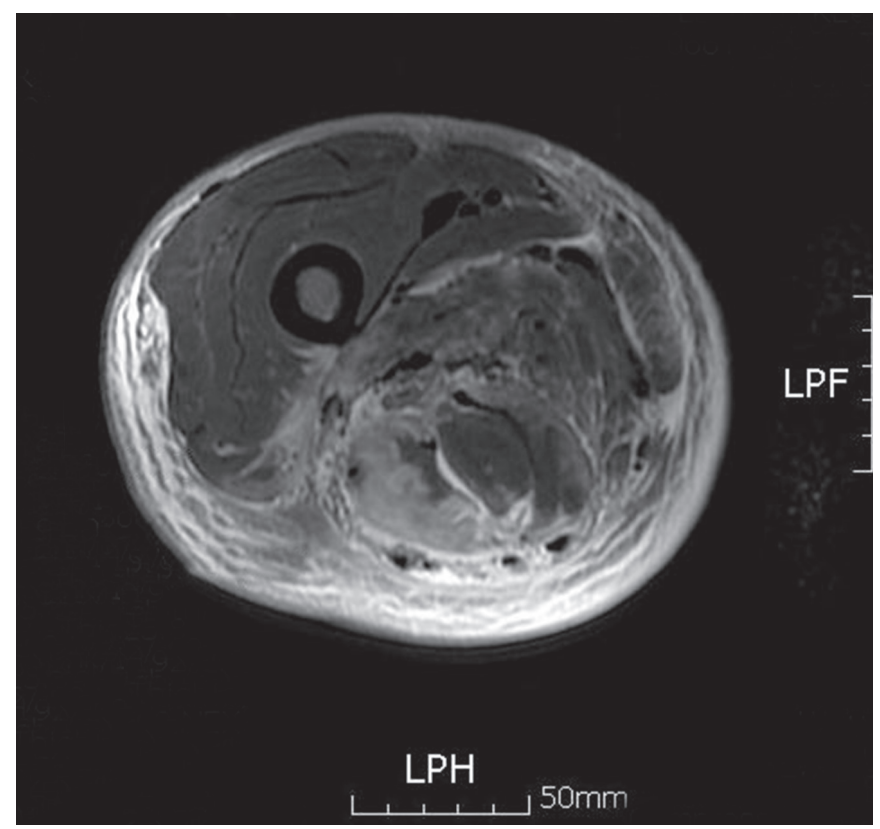

Fig. 1. T2 weighted thigh magnetic resonance imaging showing dark signal intensity air bubbles along the fascia in the posteior compartment and subcutaneous layer edema. F, feet; $\mathrm{H}$, head; L, left; P, posterior. cia in the exterior section of the right femoral regions, an edema in surrounding soft tissues, and fascial gas were observed (Fig. 1). Resection of extensive necrotic tissues and dissection of epidermis and subcutaneous tissues were immediately carried out, and the abscesses were discharged by inserting drainage tubes. Ceftriaxone, clindamycin, and amikacin were administered as initial antibiotics against all gram-positive, gram-negative, and anaerobic bacteria. Then, the necrotic tissues were repeatedly resected and washed. Enterococcus senstive to Bacteroides thetaiotaomicron

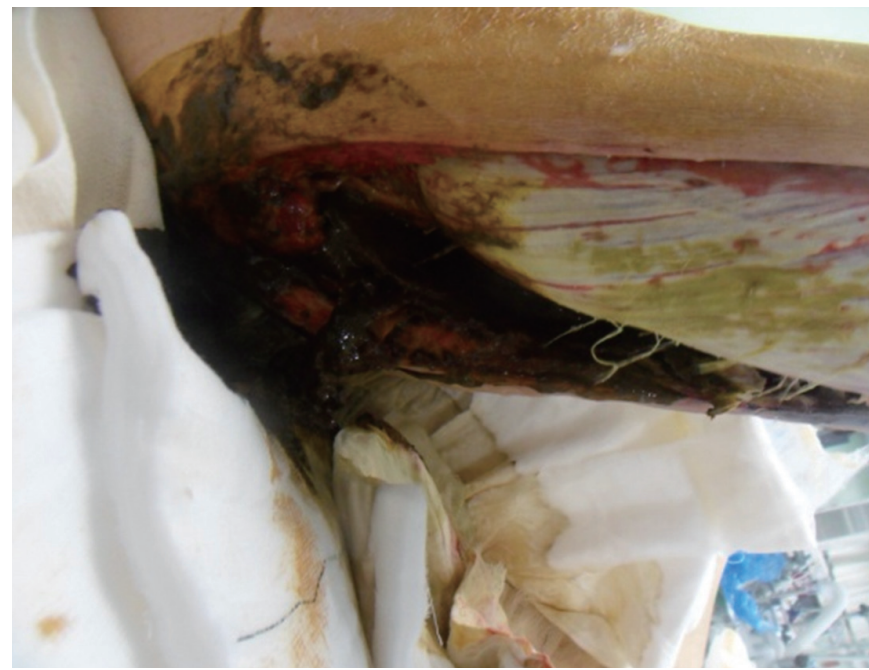

Fig. 2. Feces passing through the fistula of the right thigh.

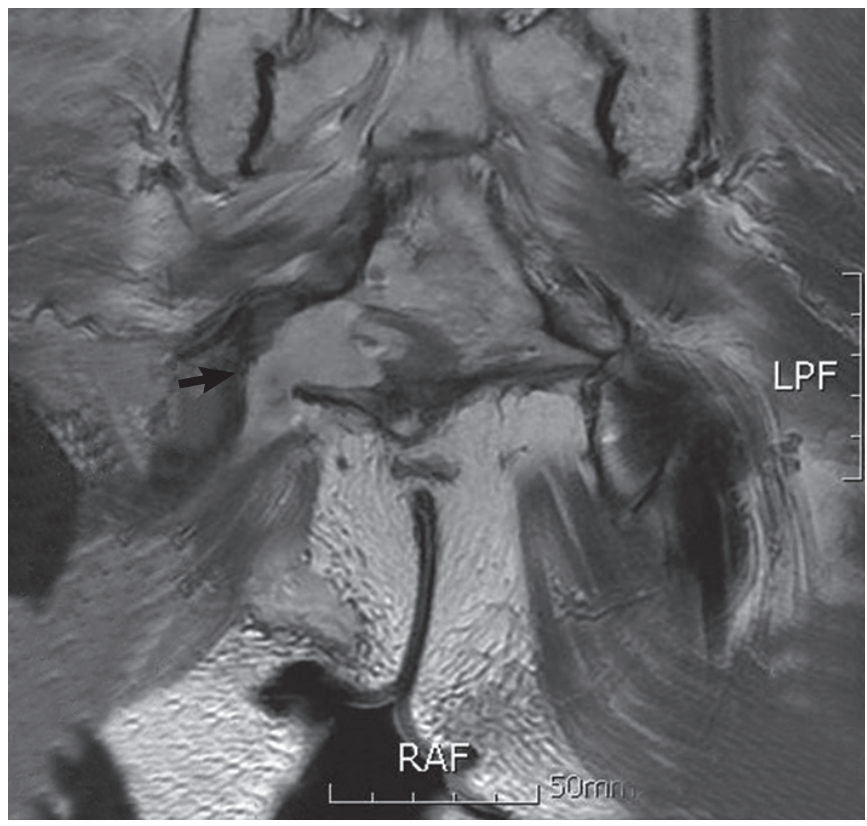

Fig. 3. T2 weighted rectal magnetic resonance imaging showing presacral abscess formation and its contiguous extension to the posterior thigh (arrow). A, anterior; F, feet; L, left; P, posterior; R, right. 


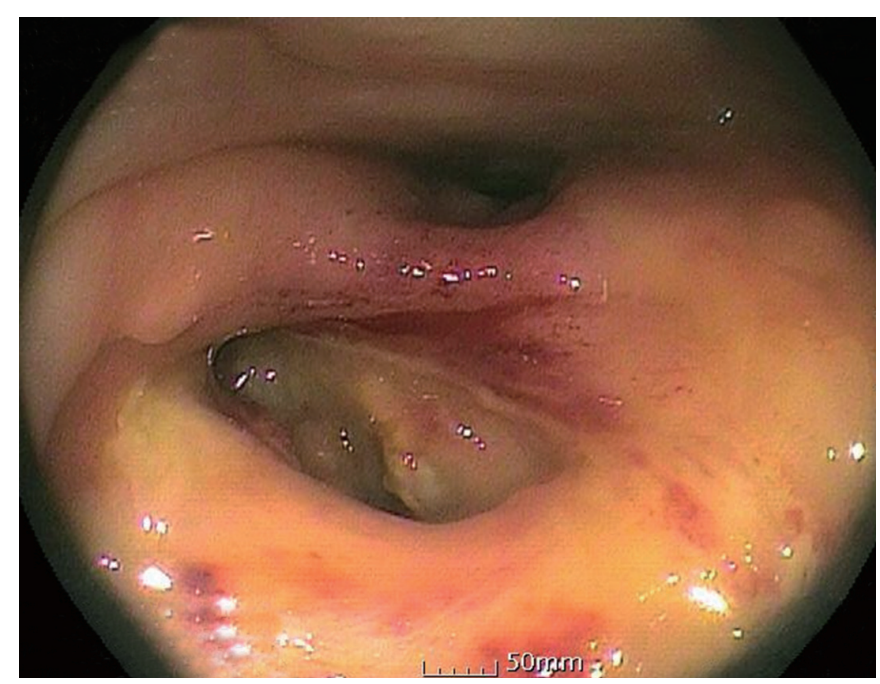

Fig. 4. Sigmoidoscopy finding showing drainage of yellowish pus through the rectal fistula.

and vancomycin were identified in the blood and necrotic lesions culture tests, respectively. Thus, the antibiotics were replaced with meropenem and vancomycin. At the same time, electrolytes were corrected, and fluids were replenished. Five days after the fascial resection, excrement was detected in the lesions of the right femoral regions (Fig. 2), and MRI was carried out in the rectal area because the presence of a rectal fistula was suspected. Pus of $8 \mathrm{~cm}$ in diameter had formed in the presacral space, and it was connected to the right gluteus maximus muscle (Fig. 3). Sigmoid endoscopy was implemented in order to confirm the rectal fistula. We were able to observe that the pus was discharging from a gap between the fascia resected region and the rectum (Fig. 4). In the colonoscopy performed two months earlier, a severe ulcer with edema and rubefaction had been observed in the connected region between the rectum and the sigmoid colon (Fig. 5). Since radiation colitis and rectal perforation were considered at that time, we suggested that the patient undergo an ileostomy, but he refused. The rectal perforation was thought to have been caused by a conventional ulcer in the anastomosis region. In order to treat the necrotizing fasciitis radically, we recommended one more time that the patient undergo an ileostomy, but he refused again. In the bacteria cultures performed on the wound, as Klebsiella pneumonia resistant to carbapenem and Enterococcus faecium resistant to multiresistant Acinetobacter baumannii and vancomycin were identified, the antibiotics were replaced with Tigecycline. Although tigecycline was administered for 16 days, 30 days after the fascial resection, the patient died due to septic shock.

\section{DISCUSSION}

Necrotizing fasciitis is a serious infection in which bacteria mainly invade subcutaneous soft tissues and rapidly spread out with deep

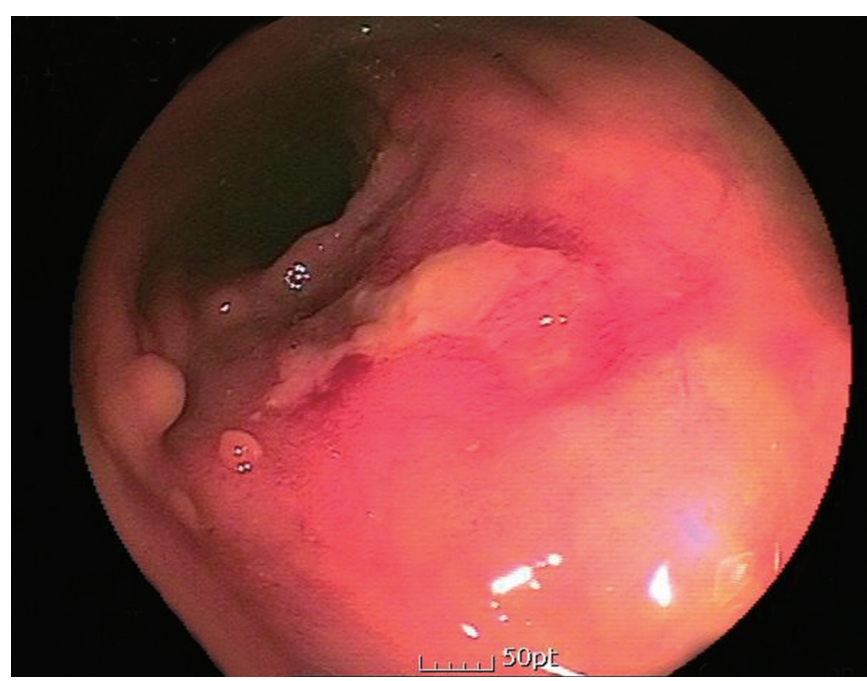

Fig. 5. Sigmoidoscopy finding showing an ulceration with edema and hyperemia around the anastomosis site.

fascia. Early diagnosis is very important due to the high mortality rate. However, as oppressive pain, rubefaction, and edema can also occur without necrosis of skin tissues in the early stage, differentiating between cellulitis, abscesses, etc. is difficult [7]. Necrotizing fasciitis can occur anywhere in the body, and it commonly takes place in the limbs, with the pelvic limb being the most common place for necrotizing fasciitis to occur. It also takes place in abdominal walls, perianal regions, inguinal regions, and surgical wounds [2]. Rarely is a case of gas gangrene caused by basic enteropathy reported, and those that are mostly originate from the large intestine [8]. The causes of death in necrotizing fasciitis are septicemia, abnormalities of blood clotting, acute renal failure, acute lung failure, multiple organ dysfunction, etc. [9].

Necrotizing fasciitis is classified into two types: Type 1 includes Streptococcus and mainly occurs in the abdominal and the inguinal regions due to a mixed infection of gram-negative rods, anaerobic bacteria, etc. Type 2 is a monomicrobial infection in which serotype A Streptococcus is the main bacteria; it is unique because fasciitis is observed in the limbs [10]. The present case was considered to be combined necrotizing fasciitis: Type 1 microbiologically because the bacteria identified in the present case were anaerobic bacteria originating from the intestine and enteric bacteria and Type 2 because it was found in the femoral regions. So far, very limited studies have investigated necrotizing fasciitis occurring in the femoral regions and associated with colorectal cancers. Takakura et al. [3] reported a case of necrotizing fasciitis in the femoral regions, complicated by an exterior pelvic abscess, in a patient with sigmoid colon cancer. In addition, Highton et al. [4] investigated a case of necrotizing fasciitis in the femoral regions that had been complications due to a piriformis muscle cause by a rectal-cancer perforation, and Liu et al. [5] reported a case of necrotizing fasciitis, caused by a rectal-cancer perforation, on both sides of the pel- 
vic limb. Although not in the femoral regions, Boo et al. [6] investigated a case, similar to the present case, of necrotizing fasciitis in the perineal region that had occurred after surgery and radiation therapy in a patient with colorectal cancer.

If necrotizing fasciitis is to be treated, necrotic tissues should be resected extensively as early as possible and reobservation of the lesions and detachment of the necrotic tissues should be carried out repeatedly. A wide range of antibiotics against all gram-positive, gram-negative and anaerobic bacteria should be administered at the time of diagnosis. Then, the antibiotics should be replaced with others that are more sensitive based upon the results of repeated bacteriologic examination. Also, electrolyte imbalance should be corrected, and sufficient fluid therapy should be carried out. Antimicrobial monotherapy does not lead to good results in necrotizing fasciitis because antibiotics are inhibited from penetrating into tissues due to the formation of microcirculation in the blood clots around lesions; in this case, the necrotic tissues had to be resected completely.

Most cases of acute radiation colitis in the early stages are temporary and can be easily treated [11] whereas chronic damage in the late stage occurs in 6 months, generally, several years, and even 30 years after the radiation therapy [12]. The symptoms start with bloody diarrhea, abdominal pain, and tenesmus, and more serious problems, including ulcers, bleeding, stenosis, perforation etc., can occur. A radiation dose of more than 5,000 cGY is generally known to cause the development of digestive tract complications [13]. Besides the radiation dose, systemic diseases, including diabetes, hypertension, and cardiovascular diseases that can cause vascular lesions, may also be causes of digestive tract damage [14]. Furthermore, chemotherapy is a factor that aggravates digestive tract complications, and many anticancer drugs are known to increase the effects of radiation [15]. In the present case, the patient, who had diabetes and rectal cancer, had damage in the digestive tract due to causes such as rectal cancer surgery, chemotherapy, and radiation therapy at a high dose (more than 5,000 cGY). Also, chronic radiation proctitis had occurred in the surgical anastomotic region, and a rectal fistula had formed due to ulcer perforation, indicating that necrotizing fasciitis might have taken place. Abdominal infection spreads to the femoral regions via various pathways: anterior or medial femoral infections via the psoas major muscle, obturator foramen, and femoral sheath, and exterior femoral infections via the sacrosciatic notch. In the present case, colonic contents were considered to have reached the femoral regions directly through the fistula after the rectal perforation, thereby causing necrotizing fasciitis. Additionally, resistance against the antibiotics developed very quickly; initially, enterococcus sensitive to $B$. thetaiotaomicron and vancomycin was identified, so the patient was treated with meropenem and vancomycin. After 2 weeks, $K$. pneumonia resistant to carbapenem and E. faecium resistant to multi-resistant $A$. baumannii and vancomycin were identified.

In conclusion, the diagnosis of necrotizing fasciitis in the patient with radiation colitis was delayed in the present case because no symptoms, such as fever or rubefactions, that would have indicated the infection, existed, even though the patient had complained of pains in the femoral regions and pelvis a month earlier. Therefore, if patients who receive rectal cancer surgeries and have radiation colitis claim to be experiencing pains in the pelvis or femoral regions, that the possibility of secondary complications due to rectal perforation inducing necrotizing fasciitis in the femoral regions should be considered. Furthermore, if necrotizing fasciitis primarily takes place in the femoral regions without skin damage, or if intestinal microflora, enterococci, etc. are detected in the wound culturing, examinations such as abdominal computed tomography should be carried out because those organisms might possibly have originated from the intraperitoneal cavity or the intestine.

\section{CONFLICT OF INTEREST}

No potential conflict of interest relevant to this article was reported.

\section{REFERENCES}

1. File TM Jr, Tan JS, DiPersio JR. Group A streptococcal necrotizing fasciitis. Diagnosing and treating the "flesh-eating bacteria syndrome”. Cleve Clin J Med 1998;65:241-9.

2. Casali RE, Tucker WE, Petrino RA, Westbrook KC, Read RC. Postoperative necrotizing fasciitis of the abdominal wall. Am J Surg 1980;140:787-90.

3. Takakura Y, Ikeda S, Yoshimitsu M, Hinoi T, Sumitani D, Takeda $\mathrm{H}$, et al. Retroperitoneal abscess complicated with necrotizing fasciitis of the thigh in a patient with sigmoid colon cancer. World J Surg Oncol 2009;7:74.

4. Highton L, Clover J, Critchley P. Necrotising fasciitis of the thigh secondary to a perforated rectal cancer. J Plast Reconstr Aesthet Surg 2009;62:e17-9.

5. Liu SY, Ng SS, Lee JF. Multi-limb necrotizing fasciitis in a patient with rectal cancer. World J Gastroenterol 2006;12:5256-8.

6. Boo YJ, Min BW, Um JW, Moon HY. Necrotizing fasciitis of perineum after surgery and radiation therapy for rectal cancer. J Korean Soc Coloproctol 2004;20:333-6.

7. Lille ST, Sato TT, Engrav LH, Foy H, Jurkovich GJ. Necrotizing soft tissue infections: obstacles in diagnosis. J Am Coll Surg 1996; 182:7-11.

8. Monneuse O, Gruner L, Barth X, Malick P, Timsit M, Gignoux B, et al. Gas gangrene of the abdominal wall due to underlying GI pathology: seven cases. J Chir (Paris) 2007;144:307-12.

9. Wong $\mathrm{CH}$, Chang HC, Pasupathy S, Khin LW, Tan JL, Low CO. Necrotizing fasciitis: clinical presentation, microbiology, and determinants of mortality. J Bone Joint Surg Am 2003;85:1454-60.

10. Chelsom J, Halstensen A, Haga T, Hoiby EA. Necrotising fasciitis due to group A streptococci in western Norway: incidence and clinical features. Lancet 1994;344:1111-5.

11. Montana GS, Fowler WC. Carcinoma of the cervix: analysis of bladder and rectal radiation dose and complications. Int J Radiat 
Oncol Biol Phys 1989;16:95-100.

12. Gilinsky NH, Burns DG, Barbezat GO, Levin W, Myers HS, Marks IN. The natural history of radiation-induced proctosigmoiditis: an analysis of 88 patients. Q J Med 1983;52:40-53.

13. Piver MS, Lele S. Enterovaginal and enterocutaneous fistulae in women with gynecologic malignancies. Obstet Gynecol 1976;48: 560-3.
14. Anseline PF, Lavery IC, Fazio VW, Jagelman DG, Weakley FL. Radiation injury of the rectum: evaluation of surgical treatment. Ann Surg 1981;194:716-24.

15. Donaldson SS, Glick JM, Wilbur JR. Letter: Adriamycin activating a recall phenomenon after radiation therapy. Ann Intern Med 1974;81:407-8. 